\title{
Diacronie
}

Studi di Storia Contemporanea

$\mathrm{N}^{\circ} 31,3$ | 2017

"Le armi della politica, la politica delle armi"

\section{Maria Teresa Giusti, La campagna di Russia 1941-1943}

\section{Edoardo Grassia}

\section{(2) OpenEdition}

\section{Journals}

\section{Edizione digitale}

URL: http://journals.openedition.org/diacronie/6315

DOI: $10.4000 /$ diacronie.6315

ISSN: 2038-0925

\section{Editore}

Association culturelle Diacronie

\section{Notizia bibliografica digitale}

Edoardo Grassia, «Maria Teresa Giusti, La campagna di Russia 1947-1943 », Diacronie [Online], №31, 3 | 2017, documento 7, online dal 29 octobre 2017, consultato il 10 décembre 2020. URL : http:// journals.openedition.org/diacronie/6315; DOI : https://doi.org/10.4000/diacronie.6315 


\section{Diacronie}

Studi di Storia Contemporanea

$31,3 / 2017$

"Le armi della politica, la politica delle armi". Ideologie di lotta ed esperienze di guerra

\section{RECENSIONE: Maria Teresa GIUSTI, La campagna di Russia 1941- 1943, Bologna, Il Mulino, 2016, 375 pp.}

A cura di Edoardo GRASSIA

Per citare questo articolo:

GRASSIA, Eduardo, «RECENSIONE: Maria Teresa GIUSTI, La campagna di Russia, 1941-1943, Bologna, il Mulino, 2016, 375 pp.», Diacronie. Studi di Storia Contemporanea : "Le armi della politica, la politica delle armi". Ideologie di lotta ed esperienze di guerra, 31, 3/2017, 29/10/2017,

URL: <http://www.studistorici.com/2017/10/29/grassia_numero_31/ >

Diacronie Studi di Storia Contemporanea $\rightarrow$ http://www.diacronie.it Rivista storica online. Uscita trimestrale.

redazione.diacronie@hotmail.it

Comitato di direzione: Naor Ben-Yehoyada - João Fábio Bertonha - Christopher Denis-Delacour - Maximiliano Fuentes Codera Anders Granås Kjøstvedt - John Paul Newman - Deborah Paci - Niccolò Pianciola - Spyridon Ploumidis - Wilko Graf Von Hardenberg

Comitato di redazione: Jacopo Bassi - Luca Bufarale - Gianluca Canè - Fausto Pietrancosta - Alessandro Salvador - Matteo Tomasoni Diritti: gli articoli di Diacronie. Studi di Storia Contemporanea sono pubblicati sotto licenza Creative Commons 3.0. Possono essere riprodotti e modificati a patto di indicare eventuali modifiche dei contenuti, di riconoscere la paternità dell'opera e di condividerla allo stesso modo. La citazione di estratti è comunque sempre autorizzata, nei limiti previsti dalla legge. 


\title{
7/ RECENSIONE: Maria Teresa GIUSTI, La campagna di Russia 1941-1943, Bologna, Il Mulino, 2016, 375 pp.
}

\author{
A cura di Edoardo GRASSIA
}

La campagna militare italiana in Russia durante il secondo conflitto mondiale è stata oggetto di pubblicazioni sin dal momento in cui i militari italiani salirono sulle tradotte per essere trasferiti su quel fronte. I primi scritti sull'argomento - quelli di regime - risalgono al 1941, con il giornale «L'Illustrazione Italiana» ${ }^{1}$ che dedicò diversi numeri al Corpo di Spedizione Italiano in Russia (CSIR). Queste pubblicazioni periodiche fecero da cornice a testi come Il bolscevismo contro Dio, contro la famiglia ${ }^{2}$ di Mario Parodi, che cercava dubbie motivazioni atte a giustificare l'intervento militare nella steppa, e Due anni di guerra. Cronache sotto gli auspici del Ministero della cultura popolare, che il Minculpop fece scrivere a più mani da improbabili inviati di guerra ${ }^{3}$. Da allora, tra monografie, raccolte di atti, diari e memorie, articoli su giornali e riviste, film, relazioni e tesi di laurea, sono stati realizzati oltre 1.700 documenti divulgativi. I libri, numericamente maggiori rispetto alle altre forme di diffusione, che direttamente o indirettamente hanno trattato l'argomento, considerando solo gli ultimi quindici anni, sono più di $500^{4}$. In tale contesto poteva risultare difficile proporre una ricerca innovativa e fornire nuovi spunti di riflessione, meritevoli di ulteriori approfondimenti. Possiamo anticipare, da subito, che Maria Teresa Giusti, con il suo La campagna di Russia 1941-19435', edito da Il Mulino nel 2016, c'è riuscita.

Concentrandoci ancora sul panorama editoriale, dalla struttura e dalla lettura del testo qui discusso, notiamo - e questo, purtroppo, non possiamo darlo per scontato - che ci troviamo di fronte ad una proposta di testo storico scritto da una storica, a differenza dell'ampia produzione maggioritaria per quanto concerne questo argomento - di appassionati o di coloro che hanno voluto scrivere, con più o meno enfasi, le proprie memorie. Questo si riscontra nella complessità

\footnotetext{
${ }^{1}$ Cfr. L'Illustrazione Italiana, n. 34 e 52 del 1941.

2 PARODI, Mario, Il bolscevismo contro Dio, contro la famiglia, Roma, Istituto Romano di Arti Grafiche di Tomminelli, 1941.

${ }^{3}$ Cronache della guerra sotto gli auspici del Ministero della cultura popolare, Roma, Istituto Romano di Arti Grafiche di Tomminelli, 1942.

${ }^{4}$ I dati sono stati elaborati dal recensore, sulla base di quelli pubblicati sul sito ufficiale dell'Unione Nazionale Italiana Reduci di Russia (UNIRR), disponibili al sito internet, URL: < www.unirr.it > [consultato il 2 maggio 2017].

${ }^{5}$ GIUSTI, Maria Teresa, La campagna di Russia 1941-1943, Bologna, Il Mulino, 2016.
} 
del lavoro svolto per il quale l'autrice ha integrato la principale bibliografia esistente, più recente e valida, con una importante ricerca archivistica sulla quale appare necessario soffermarci: Archivio Centrale dello Stato, Archivio dell'Ufficio Storico dell'Esercito, Archivio Storico del Ministero degli Affari Esteri, quello diaristico di Pieve Santo Stefano e quello dell'Istituto Campano per la Storia della Resistenza, solo per quanto concerne la parte di ricerca svolta in Italia, per passare poi al National Archives di Londra, ma soprattutto all'Archivio Statale della Federazione Russa, all'Archivio Centrale del Servizio Federale di Sicurezza della Federazione Russa, all'Archivio Centrale del Ministero della Difesa russo per quanto concerne la parte di ricerca svolta fuori confini nazionali. $\mathrm{E}$, in questa elencazione, rischiamo sicuramente di averne omesso qualcosa. Appare evidente che, oltre alle curiose scoperte che possono emergere - "la prima battaglia difensiva sul Don" della storiografia italiana, ad esempio, diviene, anche se con margini temporali non coincidenti, la "battaglia sul Volga" per la storiografia russa ${ }^{6}-$ le fonti russe sono state basilari per far luce su particolari inediti e per comprendere il punto di vista dei sovietici, il loro atteggiamento verso la guerra e i nemici, nonché verso il regime di Stalin ${ }^{7}$. E in questo, ancora, scorgiamo un importante particolare: l'autrice, conoscendo la lingua russa, ha potuto tradurre personalmente la documentazione analizzata, leggendola, quindi, con gli occhi di una storica.

Maria Teresa Giusti insegna storia contemporanea presso l'Università "Gabriele d'Annunzio" di Chieti - e si occupa da tempo di studi, ricerche e traduzioni sulla seconda guerra mondiale concentrando la sua attenzione sul fronte russo. Sono noti i suoi studi sui Prigionieri italiani in Russia ${ }^{8}$ che, oltre ad essere un punto di riferimento nell'ambito bibliografico sul tema, hanno costituito, e continuano probabilmente ad essere, un'esperienza molto importante e coinvolgente dal punto di vista professionale. Ma proprio quella importante ricerca, a nostro parere, richiedeva un passo indietro temporale, una base per meglio osservare e comprendere le vicende di quei prigionieri: lo studio sulla campagna di Russia ${ }^{9}$ fornisce proprio quel punto di partenza storico.

L'autrice parte da lontano, dalla descrizione della situazione politica post-Grande Guerra, per introdurre il lettore agli avvenimenti dei mesi precedenti a quel patto Molotov-Ribbentrop che il ministro degli esteri Galeazzo Ciano descrisse come «un colpo da maestri» dei tedeschi, che

\footnotetext{
${ }^{6}$ Ibidem, p. 214.

${ }^{7}$ Ibidem, pp. 69 et seq., pp. 81 et seq., pp. 174 et seq.

${ }^{8}$ GIUSTI, Maria Teresa, I prigionieri italiani in Russia, Bologna, Il Mulino, 2014; ID., «La questione del rimpatrio dei prigionieri di guerra italiani in Unione Sovietica 1941-1946», in L'Annale IRSIFAR. Studi e ricerche, 2000, pp. 41-81; ID., «La propaganda antifascista tra i prigionieri di guerra italiani nell'Urss», in Ricerche di Storia politica, 3, 3/2000, pp. 337-364; ID., «La memorialistica sulla prigionia di Russia», in Annali del Museo Storico Italiano della guerra, 9-10-11, 2004, pp. 11-33; ID., "Gli Internati Militari Italiani nei documenti del KGB», in Ventunesimo secolo, IX, 28, 2/2012, pp. 149-174.

${ }^{9}$ ID., La campagna di Russia 1941-1943, cit.
} 
avrebbe destabilizzato la situazione europea ${ }^{10}$. Di questo, come di molte altre vicende trattate nel libro, l'autrice fornisce anche interessanti indicazioni su come le stesse siano state trattate dalla storiografia russa permettendo, così, di osservare gli avvenimenti dai diversi punti di vista.

Proprio in questo "sconvolto" contesto europeo viene quindi illustrata la posizione italiana, sia per quanto riguarda gli aspetti politici che per quelli militari, senza spingersi in profondità, ma col solo intento di far comprendere, da subito, le grosse difficoltà italiane nell'affrontare quella guerra.

L'analisi e i commenti dell'autrice si spingono oltre le già note problematiche della mancanza di materiali ed equipaggiamenti idonei per affrontare il clima russo, facendo trasparire le inefficienze e le responsabilità di alcuni uomini di vertice, come quelle del generale Cavallero, al quale, come peraltro avvenne in altre circostanze della seconda guerra mondiale ${ }^{11}$, apparve più proficuo assecondare l'idea militare di Mussolini ${ }^{12}$ che agire da comandante di un esercito in guerra.

Restando nell'ambito storico-militare, l'autrice riporta, in varie parti del testo, alcune indicazioni sulla tipologia degli armamenti in uso presso l'esercito italiano. Al di là delle sigle e dei numeri forniti, l'interesse risiede nelle particolari osservazioni proposte - ad esempio, le dotazione dell'artiglieria italiana risultavano inefficaci contro i carri armati nemici KV1 e T- $34^{13}$ e, ancor più, la dotazione dell'armamento individuale con soli 406 moschetti automatici distribuiti per tutto il contingente del $\operatorname{CSIR}^{14}$ - che, anche quando sono prive di commenti, rendono bene l'idea sulla reale situazione militare. Anche nella più breve illustrazione dell'impiego della forza armata aerea italiana ${ }^{15}$ - schierata in Russia con la denominazione di Comando Aeronautica Fronte Orientale (CAFO) - appare evidente la non disponibilità di mezzi qualitativamente e quantitativamente adeguati ${ }^{16}$. Sono dati che, seppur relativi alla componente storico-militare del

\footnotetext{
${ }^{10}$ CIANO, Galeazzo, Diario 1937-1943 (a cura di Renzo DE FELICE), Milano, BUR, 2010, p. 332.

11 «[il duce] Non crede più a Cavallero: dice che il suo ottimismo è come il canto di chi ha paura di restare solo in una stanza». Cfr. CIANO, Galeazzo, Diario 1937-1943, cit., p. 491. Nel diario del Ministro degli Esteri italiano sono molte le pagine nelle quali scrive che il generale Cavallero comunicava con «molto ottimismo» situazioni militari che, nella realtà, vedevano le forze armate italiane soccombere in maniera talvolta irreversibile.

${ }^{12} \mathrm{Si}$ veda, al proposito, anche CEVA, Lucio, Cavallero Ugo, in Dizionario Biografico degli italiani, Volume 22 (1979), URL: < http://www.treccani.it/enciclopedia/ugo-cavallero_\%28Dizionario-Biografico\%29/ > [consultato il 5 maggio 2017].

${ }^{13}$ Il KV1 era un carro armato pesante che prendeva il nome dall'allora commissario della difesa russo Kliment Vorošilov. Aveva una lunghezza di $6,75 \mathrm{~m}$, una larghezza di 3,34 e un'altezza di 2.71, per un peso complessivo di 43 t.. Il T-34 era uno dei principali mezzi corazzati in dotazione all'esercito sovietico durante la seconda guerra mondiale e tra i suoi principali punti di forza era la lunga autonomia di $465 \mathrm{~km}$.

${ }^{14}$ STATO MAGGIORE ESERCITO - UFFICIO STORICO, Le operazioni delle unità italiane al fronte russo (1941-1943), Roma, Stato Maggiore dell'Esercito - Ufficio storico, 1977, p 330, cit. in GIUSTI, Maria Teresa, La campagna di Russia 1941-1943, cit., p. 89.

${ }^{15}$ GIUSTI, Maria Teresa, La campagna di Russia 1941-1943, cit., p. 139.

${ }^{16}$ L'autrice riporta i dati di ROCHAT, Giorgio, Le guerre italiane 1935-1943. Dall'impero d'Etiopia alla disfatta, Torino, Einaudi, 2005, p. 234.
} 
testo, finiscono per uscire da questo specifico settore e comunicano al lettore il complessivo senso di inadeguatezza delle forze armate italiane, ma anche della nazione che le avrebbe dovute sostenere.

L'arruolamento volontario-ideologico, o quello coscritto, il trasferimento al fronte con lunghi viaggi stipati nelle tradotte, la vita nella prima linea o nelle retrovie, i partigiani russi, i rapporti con i civili e molti altri nodi cruciali del libro come la ritirata, vengono illustrati citando spesso gli scritti di coloro che vissero in prima persona quegli avvenimenti, sia dalla parte italiana che da quella russa. In tal modo, oltre al puro aspetto storico, il testo trasmette sentimenti e stati d'animo di chi visse in prima persona quelle tremende esperienze ${ }^{17}$. Per queste citazioni derivanti dai diari e ancor più dalla memorialistica scritta ex post e per il loro utilizzo, bisogna tenere a mente che le memorie sui grandi eventi storici risentono inevitabilmente della psicologia individuale e tendano quindi a "drammatizzare" i fatti ${ }^{18}$, mentre la storiografia moderna, intesa come scienza, si è caratterizzata come elaborazione di metodi di indagine in grado di andare al di là delle memorie individuali ${ }^{19}$. I diari e le memorie utilizzate da Maria Teresa Giusti lungo lo svolgersi del testo non costituiscono affatto l'elemento strutturale, ma fungono infatti da "ricamo" alla ricerca archivistica portante.

Le vicende del CSIR prima e dell'Armata Militare Italiana in Russia (ARMIR) poi non furono limitate a ciò che accade sul Don o nell'area compresa tra questo è il Dnepr o attorno alla città di Stalingrado. Come messo in evidenza da Maria Teresa Giusti, la campagna di Russia si svolse anche lontano dai campi di battaglia. Il puntuale riferimento al diario di Galeazzo Ciano e al suo confronto critico con quello del generale Cavallero $^{20}$, il carteggio tra Hitler e Mussolini ${ }^{21}$, le circolari emanate dallo Stato Maggiore del Regio Esercito $^{22}$ e le informative della Polizia Politica ${ }^{23}$, ma anche gli ordini di Stalin ${ }^{24}$ non hanno il solo scopo di migliorare ed estendere la comprensione degli avvenimenti in Russia ma danno alla ricerca un giusto ampio respiro.

\footnotetext{
${ }_{17}$ «Nell'ambito delle memorie relative alla seconda guerra mondiale le testimonianze sulla campagna di Russia e sulla prigionia hanno di gran lunga superato per quantità quelle dedicate agli altri fronti [...] Memorie e diari sono una fonte importante per questo libro, specialmente quando danno conto della varietà delle percezioni dei loro autori», GIUSTI, Maria Teresa, La campagna di Russia 1941-1943, cit., p. 7.

${ }^{18}$ FUSSEL, Paul, La Grande Guerra e la memoria moderna, Bologna, Il Mulino, 1984, p. 263.

${ }^{19}$ RINALDI, Giuseppe, Storia e Memoria, in ZIRUOLO, Luciana (a cura di), I luoghi, la storia, la memoria, Genova, Le Mani, 2007. Il saggio, riveduto e ampliato, disponibile anche all'URL:

< http://digilander.libero.it/education/dati_box/ARTICOLI/Storia\%20e\%20memoria_21.pdf > [consultato il 5 maggio 2017].

${ }^{20}$ GIUSTI, Maria Teresa, La campagna di Russia 1941-1943, cit., p. 132

${ }^{21}$ Importanti sono i riferimenti al testo Hitler e Mussolini. Lettere e documenti, Milano, Rizzoli, 1946, da cui Maria Teresa Giusti ha potuto trarre importanti indicazioni sui rapporti e le richieste degli anni 1941-1943.

${ }^{22}$ Importante riferimento per gli studi che trattano questo argomento risultano essere le pubblicazioni dello STATO MAGGIORE DELL'ESERCITO - UFFICIO STORICO, L'Italia nella relazione ufficiale sovietica sulla seconda guerra mondiale, Roma, Ufficio storico SME, 1978; ID., Le operazioni delle unità italiane al fronte russo. 1941-1943, Roma, Ufficio storico SME, 2000.

${ }^{23}$ GIUSTI, Maria Teresa, La campagna di Russia 1941-1943, cit., p. 116.

${ }^{24}$ Di particolare interesse l'ordine 227, del 28 luglio 1942, «non un passo indietro!», su cui l'autrice si
} 
Analogo discorso secondo il quale la campagna di Russia non fu soltanto un evento militare, trova applicazione nella parte di testo - la seconda, in termini di lunghezza - in cui l'autrice, forte delle ricerche realizzate negli archivi russi, illustra il pensiero dei soldati italiani e di quelli russi verso la guerra e come entrambi gli schieramenti impararono a conoscersi oltre le divise. La possibilità di visionare e tradurre in prima persona la documentazione custodita nell'ex Unione Sovietica permette all'autrice di aprire spazi che difficilmente si riescono a rintracciare in altri testi, con riferimenti specifici che spostano l'attenzione dal campo militare a quello sociale: il patriottismo, le donne e la popolazione civile, fino al sesso consumato nelle case di tolleranza e ai crimini di guerra. Interessante parentesi a cui l'autrice, chiaramente, non può che dedicare singoli paragrafi ${ }^{25}$ ma che stimolano l'attenzione e la curiosità, soprattutto considerando che alcuni degli avvenimenti di cui il testo rende conto si sono perpetrati ben oltre la conclusione della seconda guerra mondiale ${ }^{26}$.

Una figura molto presente in questo volume è quella del generale Giovanni Messe ${ }^{27}$. Assieme a Mussolini, Hitler e Stalin, è il nome più ricorrente e con il suo La guerra sul fronte russo ${ }^{28} \mathrm{e}$ il fondo Messe presso l'Archivio Storico dello Stato Maggiore Esercito, costituisce un prezioso punto di riferimento per la storia non solo militare della campagna di Russia. Sembra essere anche una figura particolarmente apprezzata dall'autrice nel suo ruolo di comandante militare, un apprezzamento che si riscontra comunque in molti degli scritti sul tema qui trattato ${ }^{29}$.

Lo studio si conclude con la fine temporale della spedizione italiana in Russia, da cui il capitolo dall'eloquente titolo Il disastro dell'Armir che risulta il più lungo del libro. L'autrice introduce l'argomento, prima ancora che nei campi di battaglia, con le decisioni e gli ordini dei due principali contendenti, Hitler e Stalin, che fecero diventare quello meridionale, proprio dove era

sofferma. Cfr. GIUSTI, Maria Teresa, La campagna di Russia 1941-1943, cit., pp. 153, 210.

${ }^{25}$ GIUSTI, Maria Teresa, La campagna di Russia 1941-1943, cit., capitolo V, pp. 159, 208.

${ }^{26}$ È il noto caso del rimpatrio, a scaglioni, di prigionieri italiani trattenuti in Russia fino al 1954, ovvero dopo la morte di Stalin (5 marzo 1953). Cfr. GIUSTI, Maria Teresa, La campagna di Russia 1941-1943, cit., pp. 203-204.

${ }^{27}$ Il 17 luglio 1941 il generale Messe fu inviato a comandare il Corpo di Spedizione Italiano in Russia, per sostituire il generale Zingales, ammalato. Con il passaggio dal CSIR all'ARMIR, sconsigliato da Messe che era contrario all'invio di ulteriori grandi unità di fanteria appiedate, il comando d'armata fu affidato al generale Gariboldi. I rapporti tra i due si deteriorarono presto. Il generale Messe richiese e ottenne di rientrare in Italia il $1^{\circ}$ novembre $1942 \mathrm{e}$, successivamente, sarà inviato al confine con la Tunisia per il comando della I Armata.

${ }^{28}$ MESSE, Giovanni, La guerra sul fronte russo. Il corpo di spedizione italiano in Russia CSIR, Milano, Mursia, 2005 [ed. or. Milano-Roma, Rizzoli, 1947].

${ }^{29}$ Si vedano, tra gli altri, IGNONE, Marcello, Giovanni Messe. L'uomo, il soldato, Brindisi, Editrice Alfeo, 1992; Il Maresciallo d'Italia Giovanni Messe. Guerra, Forze Armate e politica nell'Italia del Novecento, Lecce, Congedo, 2003; LONGO, Luigi Emilio, Giovanni Messe. L'ultimo Maresciallo d'Italia, Piedimonte Matese, Imago Media per lo Stato Maggiore dell'Esercito - Ufficio Storico, 2006; OSTI GUERRAZZI, Amedeo, Noi non sappiamo odiare. L'esercito italiano tra fascismo e democrazia, Torino, Utet, 2010; LEGGIERO, Antonio, Apocalisse nella steppa. Storia militare degli italiani in Russia 1941-1943, Bologna, Odoya, 2013; VALORI, Aldo, La campagna di Russia, Roma, Grafica Nazionale Editrice, 1951; ROCHAT, Giorgio, Le guerre italiane 1935-1943, Milano, Einaudi, 2008. 
concentrato il grosso delle truppe italiane, il fronte principale ${ }^{30}$. Quest'ultima parte del testo è molto ricca di particolari di storia militare, con le battaglie sul Don, con i differenti rapporti del generale Messe e del generale Gariboldi nei confronti del comando tedesco e degli ordini da questi impartiti, fino al Capo di Stato Maggiore generale Cavallero che, sin dall'inizio, dal momento in cui occorreva prendere un'accorta decisione sulla partecipazione italiana e, ancora, quando la situazione al fronte vedeva l'avanzata dell'esercito russo - coscientemente o involontariamente o, probabilmente incoscientemente - comunicò al duce italiano parziali verità o errate notizie su ciò che stava realmente avvenendo ${ }^{31}$.

Il 16 dicembre 1942 l'esercito russo avviò l'operazione "piccolo Saturno", sfondò il fronte meridionale dando inizio alla disastrosa ritirata: «una anabasi, una delle peggiori esperienze nella storia militare del nostro paese, che ha segnato in maniera indelebile l'immaginario collettivo degli italiani $»^{32}$. L'autrice arricchisce la narrazione degli eventi con alcuni scritti memorialistici, concludendo con il bilancio di un'impresa giudicata «inutile»: l'Armir lasciò 95.000 uomini morti o vivi in mano ai russi e riportò a casa 30.000 tra feriti e congelati ${ }^{33}$ e ancor più elevato fu il prezzo pagato dai sovietici con oltre 8 milioni di morti nelle sole fila dell'esercito ${ }^{34}$.

Come l'autrice pone in evidenza, le conclusioni di questo conflitto non si ebbero il 31 gennaio 1943, con il congedo dell' $8^{\mathrm{a}}$ Armata dal fronte russo. I disagi psicologici di chi riuscì a tornare a casa, e le ancor più recenti notizie della fossa comune con circa duemila morti italiani rinvenuta a Gomel e su cui si è fatta luce solo dal 1992, ha aperto nuove indagini anche nell'ambito storico, legando la campagna militare italiana in Russia con un'altra tragedia, post-8 settembre 1943: quella degli Internati Militari Italiani (IMI), nome dato dalle autorità tedesche ai militari italiani rastrellati che non si vollero schierare con il Terzo Reich o con la Repubblica Sociale Italiana.

Per effetto della sua struttura manualistica il testo propone uno sguardo d'insieme sulla campagna di Russia fornendo anche stimoli all'approfondimento di alcuni aspetti o avvenimenti. A nostro avviso, sarebbe stato interessante integrare il volume con gli eventi e i personaggi riguardanti le forze armate aeree, sia italiane ${ }^{35}$ - per le quali ci ha fornito solo veloci indicazioni sulla scarsità di mezzi ${ }^{36}$ - che russe - per le quali ha già introdotto l'interessante presenza femminile nelle carlinghe di caccia e bombardieri ${ }^{37}$ - e quelli relativi agli uomini e mezzi della

\footnotetext{
${ }^{30}$ GIUSTI, Maria Teresa, La campagna di Russia 1941-1943, cit., p. 210.

${ }^{31}$ Ibidem, p. 217.

${ }^{32}$ Ibidem, pp. 249-250.

${ }^{33}$ Ibidem, p. 263.

${ }^{34}$ Per i dati statistici di morti e feriti, militari e civili, si veda ibidem, p. 272.

${ }^{35}$ Si segnala, tra l'altro, il carteggio custodito presso l'Ufficio Storico dell'Aeronautica, con particolare riferimento al fondo di Superaereo e a quello che raccoglie i Diari Storici dei Reparti impegnati nella seconda guerra mondiale.

${ }^{36}$ Vedasi nota 16.

${ }^{37}$ GIUSTI, Maria Teresa, La campagna di Russia 1941-1943, cit., p. 176.
} 
Regia Marina schierati nel Mar Nero e sul lago Ladoga ${ }^{38}$, affiancando alle figure dei generali dell'esercito quali Cavallero, Messe e Gariboldi, anche il ricordo del generale dell'aeronautica Enrico Pezzi, morto in una missione di volo per portare soccorso alle truppe a Čertkovo ${ }^{39}$, e dell'ammiraglio Francesco Mimbelli ${ }^{40}$.

Il testo è corredato di un inserto fotografico e di una appendice. Nel primo, il susseguirsi delle immagini è strutturato secondo il procedere temporale della campagna di Russia. Prende avvio con le foto della partenza, dove soldati e mezzi sfilano davanti a Mussolini e al re. Seguono poi alcune immagini del viaggio e di scene di vita quotidiana dove compaiono anche civili, donne e bambini. Vi sono istantanee di prigionieri russi e quelle legate a due elementi che risultano fondamentali in questa storia: la neve e il ghiaccio, prima, e il fango, dopo il loro scioglimento, che caratterizzarono la vita e la guerra su questo fronte. Senza giustamente ricorrere a particolari spettacolarizzazioni, compaiono anche foto delle amputazioni mediche degli arti inferiori per congelamento e, infine, la immagini della ritirata. L'inserto fotografico termina con una cartina geografica la cui presenza è sicuramente molto preziosa, ma che avremmo preferito maggiormente dettagliata e ingrandita, possibilmente su più pagine, in modo da avere uno strumento migliore per individuare località normalmente poco note o sconosciute e dai nomi talvolta di difficile lettura. L'appendice, invece, posta in chiusura del libro, raccoglie alcuni documenti originali, sia italiani che tradotti dal russo, la cui scelta è orientata principalmente a dare spunti sull'attività di intelligence dei due schieramenti, con circolari e interrogatori.

Il libro, complessivamente, ha il pregio di essere il prodotto di una importante ricerca storicoarchivistica che l'autrice ha tradotto in una piacevole narrazione storica. Pur utilizzando necessariamente anche una terminologia tecnico-militare, risulta di piacevole lettura e facile comprensione anche a chi non ha dimestichezza nel settore ma vuole acquisire una corretta conoscenza degli avvenimenti in esame che, come evidenziato ${ }^{41}$, furono probabilmente decisivi per le sorti del secondo conflitto mondiale.

\footnotetext{
${ }^{38}$ Particolare riferimento alla IV Flottiglia che operò nel Mar Nero con il fine di impedire i rifornimenti navali da sud, soprattutto in occasione dell'assedio di Sebastopoli, e della XII Squadriglia che invece si schierò nel lago Ladoga, a nord, con l'identica funzione nei confronti dell'assedio di Stalingrado.

${ }^{39} \mathrm{Il}$ generale di brigata aerea Enrico Pezzi, era subentrato al colonnello Drago il 12 febbraio 1942 al comando del CAFO e morì a seguito di incidente di volo il 29 dicembre 1942 quando decollò dalla base di Woroschilowgrad in direzione di Čertkovo, per prestare soccorso ai soldati italiani rimasti accerchiati. Con lui, tra gli altri, il colonnello medico Federico Bocchetti. Anche l'autrice riporta questa importante vicenda in nota, cfr. GIUSTI, Maria Teresa, La campagna di Russia 1941-1943, cit., p. 326, nota 39.

${ }^{40}$ L'ammiraglio Francesco Mimbelli fu il comandante della $4^{a}$ Flottiglia MAS nel Mar Nero. Promosso ammiraglio di squadra nel 1957, nel 1993 la Marina Militare decise di titolargli un cacciatorpediniere lanciamissili.

${ }^{41}$ GIUSTI, Maria Teresa, La campagna di Russia 1941-1943, cit., p. 210
} 


\section{L'AUTORE}

Edoardo GRASSIA, diploma di laurea in Sociologia e laurea in Storia medievale, moderna e contemporanea presso l'Università La Sapienza di Roma, è dottorando di ricerca in Storia dell'Europa presso lo stesso ateneo. Si è occupato dell'Aviazione Legionaria da Bombardamento, da cui è nata la pubblicazione L'Aviazione Legionaria da bombardamento (Spagna 1936-1939). Iniziare da stanotte azione violenta su Barcellona (Roma, IBN Editore, 2009) e di Resistenza e guerra di Liberazione con lo studio Sabato Martelli Castaldi. Il generale partigiano (Milano, Mursia, 2016). È autore di alcuni articoli editi da «Diacronie. Studi di Storia Contemporanea». Membro della Società Italiana di Storia Militare (SISM), svolge regolarmente attività di studio e di ricerca in ambito storico-sociale e storico-militare.

URL: < http://www.studistorici.com/progett/autori/\#Grassia > 